\title{
Intracranial Pial Arteriovenous Fistula Presenting as Brain Hemorrhage in Newborn Infants
}

Soo Yeon Kim, M.D., Ho Seon Eun, M.D., Jeong Eun Shin, M.D., Soon Min Lee, M.D., Min Soo Park, M.D., Ran Namgung, M.D., and Kook In Park, M.D.

Division of Neonatology, Department of Pediatrics, Severance Children's Hospital, Yonsei University College of Medicine, Seoul, Korea

\section{ABSTRACT}

Congenital intracranial pial arteriovenous fistulas (AVFs) are rare cerebrovascular lesions. Their clinical manifestations tend to vary according to age, with pediatric populations being more likely to have symptoms like congestive cardiac failure and seizures because of arteriovenous shunting; hemorrhage is the major presentation in adult populations. Pediatric populations, especially newborn infants, seldom experience a hemorrhagic event. Here, we report two rare cases of neonates with congenital pial AVF presenting as intraventricular and subdural hemorrhage, respectively, which were treated with endovascular embolization.

Key Words: Arteriovenous fistula, Infant, Newborn, Intracranial hemorrhages, Therapeutic embolization

\section{INTRODUCTION}

Intracranial pial arteriovenous fistulas (AVFs) are rare vascular lesions of the brain, and have distinct angioarchitectural characteristics, consisting of single or multiple arterial feeders and a drainage vein without an intervening nidus. AVFs account for $1.6-8.4 \%$ of all intracranial vascular malformations ${ }^{1-3)}$ and represent approximately $4 \%$ of pediatric cerebral vascular malformations $s^{4}$.

Clinical presentations of pial AVFs vary according to age, with pediatric populations being more likely to have symptoms like high-output cardiac failure and seizures because of arteriovenous shunting, whereas hemorrhage is the major presentation in adult patients. Pediatric patients, especially newborn infants, seldom experience a hemorrhagic event ${ }^{3,5}$.

This report describes two unusual cases of newborn infants with intracranial pial AVFs who presented with brain hemorrhage and were treated with endovascular embolization. To our knowledge, this is the first case report of pial AVFs presenting with intracranial hemorrhage (ICH) in newborn infants in Korea.
Received: 10 August 2015

Revised: 10 September 2015

Accepted: 14 September 2015

Correspondence to:

Kook In Park, M.D., Ph.D.

Division of Neonatology, Department of Pediatrics, Severance Children's Hospital, Yonsei University College of Medicine, 50-1 Yonsei-ro, Seodaemun-gu, Seoul 03722, Korea

Tel: +82-2-2228-2059

Fax: +82-2-393-9118

E-mail: kipark@yuhs.ac

Copyright(c)

By Korean Society of Neonatology.

All right reserved.

This is an Open-Access article distributed under the terms of the Creative Commons Attribution Non-Commercial License (http://creativecommons.org/licenses/ by-nc/3.0), which permits unrestricted non-commercial use, distribution, and reproduction in any medium, provided the original work is properly cited. 


\section{CASE REPORT}

\section{Case 1}

A 22-day-old male infant was transferred to the emergency department in our hospital after an episode of sudden vomiting. The infant had been uneventfully delivered from a healthy mother at 39 weeks gestational age, weighing $3.2 \mathrm{~kg}$. Initial blood pressure was $83 / 48 \mathrm{mmHg}$, with a heart rate of 152 beats per minute and respiratory rate of 40 breaths per minute. There was no history of trauma, and neurological examination was normal. However, physical examination revealed a pale appearance with a bulging anterior fontanel. There was no cardiac murmur. His initial hemoglobin level was $7.7 \mathrm{~g} / \mathrm{dL}$, and computed tomography (CT) scan of the brain revealed an extensive intraventricular hemorrhage (IVH) with hydrocephalus (Figure 1A). Emergent bilateral frontal external ventricular drain (EVD) insertion was performed. A subsequent brain CT and angiogram demonstrated an AVF with a large aneurysmal dilatation of the drainage vein. Digital subtraction angiography (DSA) revealed a pial AVF feeding from branches of the left posterior cerebral artery (PCA) and draining into a dilated venous pouch which had a stenotic connection to the transverse sinus (Figure 1B). The patient subsequently underwent endovascular embolization. After distal selection of the largest left PCA feeder with a microcatheter, $85 \%$ glue embolization was performed. A postembolization angiogram showed some fistula remains; another left PCA feeder was selected and underwent $20 \%$ glue embolization. A final angiogram showed complete occlusion of the pial AVF with an intact transverse sinus (Figure 1C). After 2 weeks, the patient underwent insertion of a ventriculoperitoneal (VP) shunt and was discharged. A CT scan of the brain performed 1 month later showed a resolved IVH with stable ventricular dimensions. On 7-month follow-up, his Bayley Scales of Infant Development-II (BSID-II) showed a Mental Development Index (MDI) of 39 and Psychomotor Development Index (PDI) of 26 , which indicated the developmental status of a 3 month old. The Gross Motor Function Measure (GMFM) score was $14.6 \%$, which represents category IV, or significant delay.

\section{Case 2}

A 30-day-old female infant presented to the emergency department after 2 hours of repeated apnea lasting nearly 10 seconds and recurring every 3 to 5 minutes. She demonstrated sudden breath-holding episodes with cyanosis and showed upward eyeball deviation with stretching of both upper limbs. The baby had been born at 36 weeks of gestation, weighing 3.2 $\mathrm{kg}$, and had no history of head trauma. Her chest radiograph showed a normal sized heart and clear lung parenchyma. Emergent brain CT performed for the evaluation of the seizurelike movements confirmed a subdural hemorrhage (SDH) in the right frontoparietal region with a large hypodense structure with internal mixed vascularity at the right occipital lobe, which was thought to be a vascular malformation (Figure 2A). Right internal carotid artery angiography identified a pial AVF feeding from the right PCA and draining to a dilated venous pouch, with a stenotic connection to the superior sagittal sinus (Figure
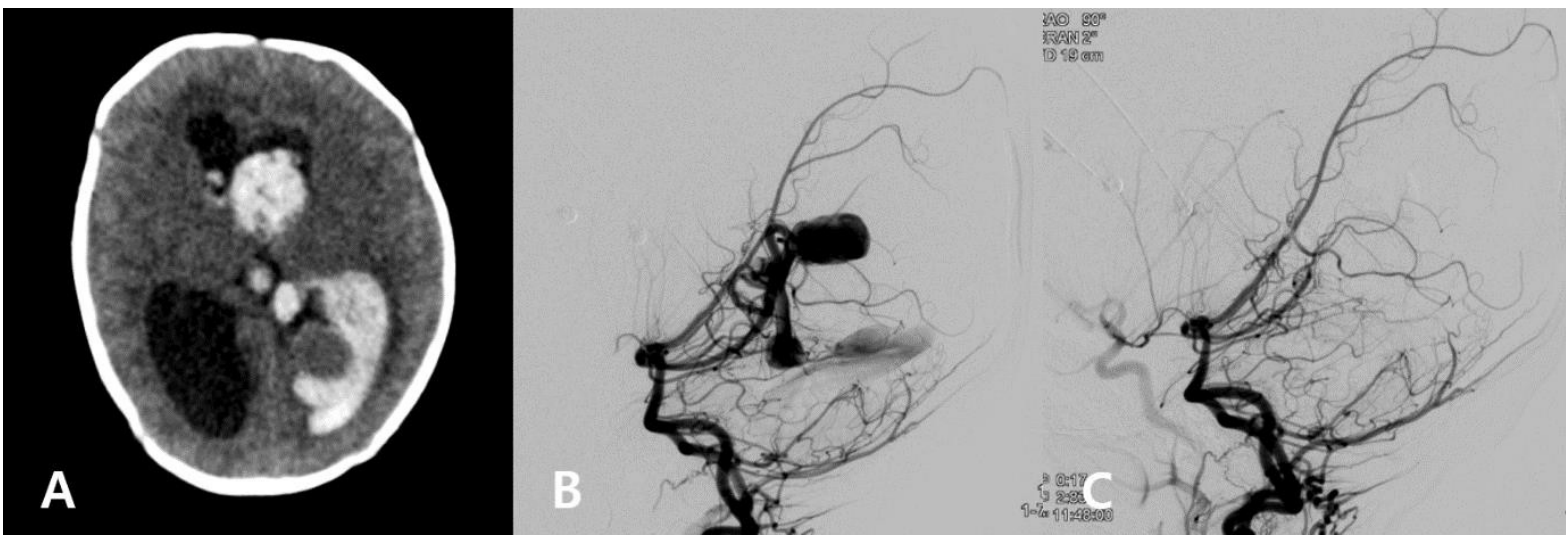

Figure 1. Findings from a 22-day-old male neonate presenting with a sudden episode of vomiting. (A) Initial computed tomography (CT) scan showing an extensive intraventricular hemorrhage with hydrocephalus. (B) Lateral projections of left vertebral angiography show a high-flow pial arteriovenous fistula fed by the left posterior cerebral artery and draining into a dilated venous pouch, which had a stenotic connection to the transverse sinus. (C) Lateral projections of postembolization left vertebral angiography showing complete occlusion of the pial arteriovenous fistula with an intact transverse sinus. 


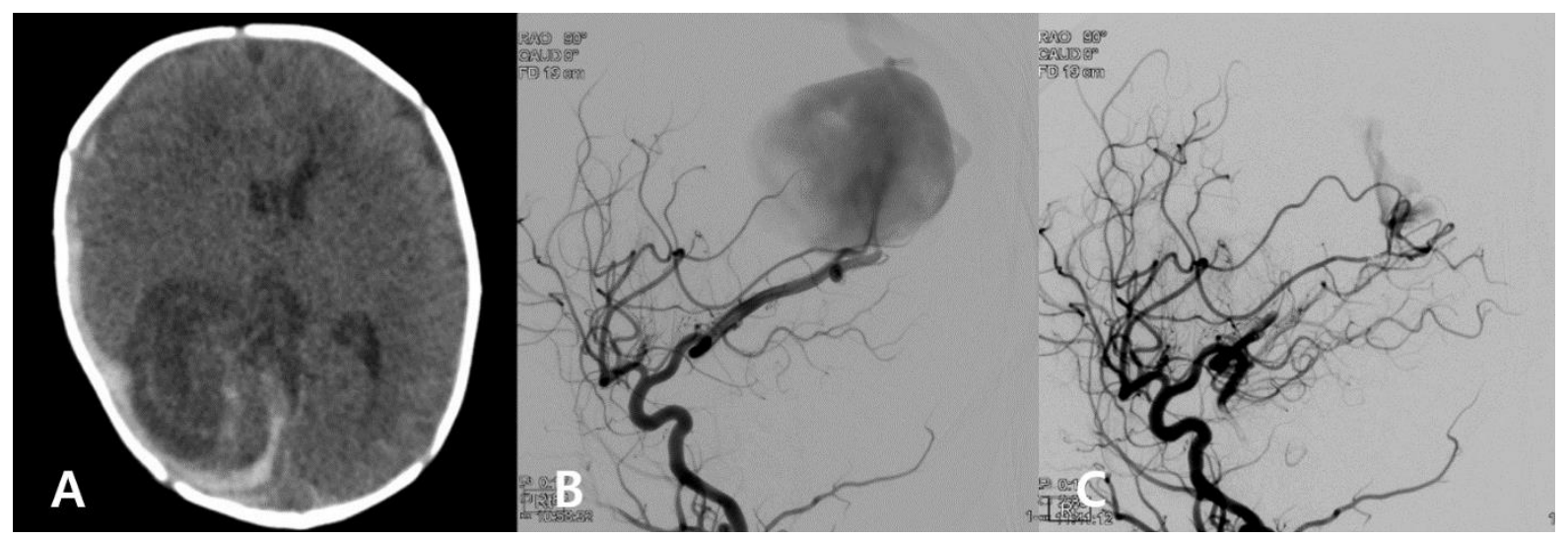

Figure 2. Findings from a 30-day-old female neonate presenting with breath-holding episodes and seizure-like movements. (A) Initial computed tomography scan showing a subdural hemorrhage in the right frontoparietal region, and a hypodense structure with internal mixed vascularity in the right occipital lobe. (B) Lateral projections of right internal carotid angiography revealing a pial arteriovenous fistula (AVF) feeding from the right posterior cerebral artery and draining into a dilated venous pouch, with a stenotic connection to the superior sagittal sinus. (C) Lateral projections of right internal carotid angiography showing near complete occlusion of the pial AVF, with an occluded posterior cerebral artery feeder but an intact middle cerebral artery and fine branch feeders.

2B). Concomitant embolization was attempted. After distal selection of the right PCA feeder, it was coiled for flow reduction and a $90 \%$ glue embolization was performed. A final angiogram showed near complete occlusion of the pial AVF, with an occluded PCA feeder but intact middle cerebral artery and fine branch feeders (Figure 2C). The infant tolerated the procedure well and was discharged two weeks later after an ultrasound exam that confirmed no newly developed hemorrhage but only an echogenic lesion, which was a thrombus-filled AVF without definite vascularity. At the 3-month follow-up examination, brain magnetic resonance imaging showed a venous pouch of decreased size, with an inner thrombus and nearly resolved SDH on the right. Two years later, a brain CT scan showed further reduction in the size of the pouch, and the hemorrhage had completely resolved. However, encephalomalacic changes of the right parietotemporal-occipital lobes led to left spastic hemiplegia. Although she did not show any definite impairment on vision and auditory testing, the Gross Motor Function Measure-88 (GMFM-88) score assessed at corrected age of 3 years and 5 months was $86.7 \%$, and the Modified Barthel Index (MBI) score was 30 , indicating severe dependence for daily activities.

\section{DISCUSSION}

Earlier, pial AVFs were often grouped with arteriovenous malformations (AVMs) in the clinical literature due to their infre- quent occurrence and the absence of a specific classification category. Advances in angiography, however, have allowed the distinction of pial AVF presenting as an abrupt transition from the arterial feeder(s) to the draining vein, without the plexiform nidus of a typical AVM. The prevalence of pial AVFs can be estimated at $0.1-1 / 100,000$, of which the majority of cases are diagnosed in the second and third decades of life. In children, they may present between the ages of 3 and 15 years. Pial AVF, however, is rarely seen in newborn infants. Although little is known regarding its etiology, it is believed that abnormal angiogenesis and associated vascular growth factors, as well as embryological missteps, may play a role in the formation of congenital pial AVFs ${ }^{6}$.

The natural history of pial AVFs is also largely unknown due to its rarity, especially in pediatric populations. According to previous reports, several distinctions have been made between the clinical features of pial AVFs in pediatric and adult populations. The pediatric type has a high percentage of symptoms related to shunting effects, such as congestive heart failure and seizures, and mass effects like macrocrania, headaches, and focal neurological deficits. On the other hand, the adult type usually presents with hemorrhage ${ }^{7,8)}$. In addition, the clinical presentation of pial AVFs tends to vary by age in pediatric patients. Hetts et al. ${ }^{5)}$ showed that this heterogeneous disease entity could be divided into two groups, based on the onset of presentation at the age of 2 years. The study demonstrated angioarchitectural findings according to age, with younger 
children being more likely to harbor large, complex, multi-hole AVFs (which is consistent with the findings in our report) that have high-flow arteriovenous shunting and consequent venous dilatation. On the contrary, older children were more likely to have a single-hole pial $\mathrm{AVF}^{5)}$ and decreased incidence of venous varices $^{7)}$. In a previous study, venous dilatation was found to be highly correlated with a decreased risk of hemorrhage; it was postulated that this dilatation might exert a buffer effect for tolerating high arterial pressure ${ }^{7}$. Given this finding, intracranial hemorrhage in newborn infants is reported to be especially $\operatorname{rare}^{3,5,8,9)}$.

This report describes rare cases of newborn pial AVFs presenting as spontaneous ICH. As previously noted, younger age or the presence of ICH at presentation may signify an unfavorable prognosis in $\mathrm{AVFs}^{8)}$. Clearly, patients who presented with ICH had a higher mortality rate than those without ICH. Younger age at diagnosis is also assumed to be a potential indicator of increased mortality. A superimposed hemorrhage on the vulnerable brain and immature hydrovenous system of newborn infants may clearly result in even worse outcomes. While little is known about the exact mechanisms of hemorrhage in pial AVFs, other studies have demonstrated the possible link between hemorrhage and pial venous outflow obstruction by venous stenosis or thrombosis ${ }^{3,10)}$. We identified such venous outflow stenosis in this report as well. Early angiographic identification of a fistula might play an important role in assessing the risk for a hemorrhage in newborn infants, but further studies are needed. In addition, considering such rare incidence of pial AVFs resulting in neonatal ICH, further studies including long-term follow up data are mandatory to elucidate its possible risk factors, pathogenesis, and outcomes.

The goal of treatment in pial AVFs is the rapid control of shunting, because spontaneous closure of the fistula cannot be expected. With advances in microcatheters and various types of embolic agents, transarterial embolization has become the firstline therapeutic choice, offering both safety and effectiveness ${ }^{11-13)}$. Other treatment options include radiosurgery and open neurovascular surgery ${ }^{14,15}$. However, the anatomical and physiological characteristics of pial AVFs in newborns and infants make it particularly difficult to treat. Challenges include distinct anatomical characteristics, such as multiple tortuous intracranial feeding arteries with small vessel calibers, with high perfusion pressure, and the immature and vulnerable characteristics of the growing brain ${ }^{5)}$. Previous research comparing the out- comes of pial AVFs between infants and older children reported that the procedural complication rate was higher in infants, as high as $60 \%^{5,8)}$. An overall mortality rate near $17 \%$ in infants treated for pial AVFs has been reported ${ }^{9)}$. Despite these difficulties, much literature recommends the urgent management of pial AVFs in newborns and infants, even when asymptomatic, considering the unfavorable natural history of the fistula ${ }^{12,16,17)}$. In our report, the patients tolerated the procedure well, but went through neurological sequelae due to the initial intracranial hemorrhage.

In conclusion, since pial AVF has a poor natural history and possesses significant morbidity and mortality secondary to rupture and bleeding, a prompt angiographic assessment and treatment is necessary in newborn infants. This report highlights the importance of considering the rare disease entity of pial AVF as a cause of spontaneous intracranial hemorrhage in newborn infants.

\section{REFERENCES}

1) Halbach VV, Higashida RT, Hieshima GB, Hardin CW, Dowd CF, Barnwell SL. Transarterial occlusion of solitary intracerebral arteriovenous fistulas. AJNR Am J Neuroradiol 1989; 10:747-52.

2) Tomlinson FH, Nichols DA, Fode NC. Arteriovenous fistulas of the brain and the spinal cord. J Neurosurg 1993;79:16-27.

3) Weon YC, Yoshida Y, Sachet M, Mahadevan J, Alvarez H, Rodesch G, et al. Supratentorial cerebral arteriovenous fistulas (AVFs) in children: review of 41 cases with 63 non choroidal single-hole AVFs. Acta Neurochir (Wien) 2005;147:17-31.

4) Cooke D, Tatum J, Farid H, Dowd C, Higashida R, Halbach V. Transvenous embolization of a pediatric pial arteriovenous fistula. J Neurointerv Surg 2012;4:e14.

5) Hetts SW, Keenan K, Fullerton HJ, Young WL, English JD, Gupta N, et al. Pediatric intracranial nongalenic pial arteriovenous fistulas: clinical features, angioarchitecture, and outcomes. AJNR Am J Neuroradiol 2012;33:1710-9.

6) Lasjaunias P. A revised concept of the congenital nature of cerebral arteriovenous malformations. Interv Neuroradiol 1997;3:275-81.

7) Yang WH, Lu MS, Cheng YK, Wang TC. Pial arteriovenous fistula: a review of literature. Br J Neurosurg 2011;25:580-5.

8) Madsen PJ, Lang SS, Pisapia JM, Storm PB, Hurst RW, Heuer GG. An institutional series and literature review of pial arteriovenous fistulas in the pediatric population: clinical article. J Neurosurg Pediatr 2013;12:344-50. 
9) Kraneburg UM, Nga VD, Ting EY, Hui FK, Lwin S, Teo C, et al. Intracranial pial arteriovenous fistula in infancy: a case report and literature review. Childs Nerv Syst 2014;30:365-9.

10) Miyasaka $Y$, Yada $K$, Ohwada $T$, Kitahara $T$, Kurata $A$, Irikura $K$. An analysis of the venous drainage system as a factor in hemorrhage from arteriovenous malformations. J Neurosurg 1992;76:239-43.

11) Newman CB, Hu YC, McDougall CG, Albuquerque FC. Balloon-assisted Onyx embolization of cerebral single-channel pial arteriovenous fistulas. J Neurosurg Pediatr 2011;7:637-42.

12) Jabbour P, Tjoumakaris S, Chalouhi N, Randazzo C, Gonzalez LF, Dumont A, et al. Endovascular treatment of cerebral dural and pial arteriovenous fistulas. Neuroimaging Clin N Am 2013; 23:625-36.

13) Zaidi HA, Kalani MY, Spetzler RF, McDougall CG, Albuquerque FC. Multimodal treatment strategies for complex pediatric cerebral arteriovenous fistulas: contemporary case series at Barrow Neurological Institute. J Neurosurg Pediatr 2015;15: 615-24.

14) Upchurch K, Feng L, Duckwiler GR, Frazee JG, Martin NA. Nongalenic arteriovenous fistulas: history of treatment and technology. Neurosurg Focus 2006;20:E8.

15) Berenstein A, Ortiz R, Niimi Y, Elijovich L, Fifi J, Madrid M, et al. Endovascular management of arteriovenous malformations and other intracranial arteriovenous shunts in neonates, infants, and children. Childs Nerv Syst 2010;26:1345-58.

16) Toma AK, Davagnanam I, Ganesan V, Brew S. Cerebral arteriovenous shunts in children. Neuroimaging Clin N Am 2013; 23:757-70.

17) Nakiri GS, Abud TG, Oliveira RS, Santos AC, Machado HR, Abud DG. Endovascular treatment of intracranial pial arteriovenous fistula. Arq Neuropsiquiatr 2010;68:463-5. 